\title{
Internet Telephony or Circuit Switched Telephony: Which is Cheaper?
}

\author{
Martin B.H. Weiss \\ (mbw+@pitt.edu) \\ Telecommunications Program \\ University of Pittsburgh \\ Pittsburgh PA 15260
}

\author{
Junseok Hwang \\ (hwang@tele.pitt.edu) \\ Telecommunications Program \\ University of Pittsburgh \\ Pittsburgh PA 15260
}

September 4, 1998

\begin{abstract}
Telephony is an Internet application that has the potential to radically alter the telecommunications environment. This application may affect traditional regulatory structures, subsidy structures, business models, etc. Today, users can transmit telephone-like voice traffic over the Internet at zero incremental price, unlike circuit switched telephony, which has a per-minute incremental price. While these economics make this a compelling application for international users in particular, this research was carried out to determine whether Internet Telephony (Itel) was a fundamentally cheaper approach to the interoffice transmission and switching of voice, or whether the price difference was the result of an implicit regulatory subsidy (i.e., ISPs are not required to pay the CCLC).

This paper is intended to be a first estimate of the switching and interoffice transmission costs for Itel and circuit switched networks. To evaluate these costs, we build a "green fields" network for an area and a population equivalent to the US state of Rhode Island $\left(\approx 1 \mathrm{M}\right.$ people in $\left.3140 \mathrm{~km}^{2}\right)$ in both technologies using "normal" engineering standards for voice quality service and compare the costs. To realize the comparable packet switched voice service with circuit switched, we dimension the Itel network to have a service quality generally similar to conventional telephony. The networks are developed in Comnet III to support the appropriate dimensioning of the networks. In this initial computation, we assume that the local loop costs are identical for both technologies, and that the services provided are identical.

We find that the switching and transmission costs for Itel are $38 \%$ lower than the costs for circuit switching. We further find that this cost difference is largely due to the reduced interoffice transmission capacity required by Itel. This is consistent with the findings in [10] and what many potential ITSPs (Internet Telephony Service Providers) ${ }^{1}$ claim[1].
\end{abstract}

\section{Introduction}

One of the most challenging developments in telecommunications in recent years has been the emergence of Internet Telephony (Itel) ${ }^{2}$. In the U.S., Internet Service Providers (ISPs) are exempt from the access charge system that is used to support local service. Internationally, ISPs are often outside of the traditional regulatory structures because they are Value Added Networks (VANs), which have historically been less regulated than providers of public switched service. Furthermore, the international accounting and settlements process is substantially challenged in the face of Itel, because the benefits of arbitrage are substantial. Even if including Itel under the normal regulatory framework was a social goal, it is far from clear how [5].

One of the questions that has arisen is whether the per call price difference between Itel and the public switched telephone network occurs because Itel fundamentally more cost effective than the traditional public

\footnotetext{
${ }^{1}$ For example Concentric Network Corp.

${ }^{2}$ We would like to thank the people at Cisco Systems, Inc., Lucent Technologies, Inc., Hyperion Telecommunications, Inc. and NPT Systems, Inc. for their time and willingness to help.
} 
switched telephone network, or if is due to a regulatory artifact ${ }^{3}$. If it is the former, it implies a pending revolution in the design, organization, and operation of the public network infrastructure. If it is the latter, Itel will be a marginal phenomenon in the long run, requiring little if any attention from public regulators ${ }^{4}$.

In this paper, we begin to address this question by constructing networks (on paper) and evaluating their costs. This is not intended to be a complete or final design, but rather one that captures the major switching and transmission cost elements of the interoffice network needed to address the question articulated in the previous paragraph. This is intended to be a "first order" analysis; there are many assumptions, the relaxation of which may result in further insights.

\section{Technological Overview}

The purpose of this section is to outline the key distinctions between these two technologies. More definitive discussions can be found elsewhere.

\subsection{Assumptions and Simplifications}

As this is a first order analysis, there are some simplifying assumptions that we wish to make. These include:

1. The focus will be on the costs of switching, signaling and trunking. Thus, we will assume that similar access and transmission technologies will be used. An actual Itel-based network might well consider alternatives to the current local loop technology. In terms of transmission, this assumption is not unreasonable, as the higher speed aggregate transmission links probably would use the same technology.

2. We will assume current technologies. We considered compressed voice and silence suppression technologies for the Itel access networks and fast IP router switches for Itel switching functions.

3. We will assume equal levels of demand for both technologies.

4. We will assume that the services are perfect substitutes for each other. That is, we will assume that the user will not be able to tell the difference between Itel and traditional telephone service from the point of view of major functions. Today, many consumers report poorer service quality with Itel as well as limitations surrounding the PC [4]. On the other hand, Itel allows a level of service integration that is difficult or costly to achieve with circuit switched telephony.

5. We will assume that neither service is subject to line charges for regulatory purposes.

6. We assume this network is only connected to similar networks. As a result, we make no allowance for gateway or interconnection facilities.

7. We assume that the cost of transmission is constant over the life of the study.

\subsection{The Public Switched Telephone Network}

The public switched telephone network has evolved into its present form over its 100 year history. The network was initially optimized to handle low bandwidth $(4 \mathrm{kHz})$ channels using manual technology (no mean feat, as illustrated by Mueller [8]). As technology evolved, so did the way in which switching was performed. The digitization of the network allowed for high speed data services. Advances in packet switching technology allowed for the transformation of the signaling network to support a wide array of enhancements to basic service. Despite these advances, the circuit switched telephone network can be characterized by the following:

1. It is capable of handling many dedicated low bandwidth channels (64kbps or $4 \mathrm{kHz})$. Adaptive Differential PCM (ADPCM) was developed to transmit "toll quality" voice over 32kbps, but this technology has not been widely installed.

\footnotetext{
${ }^{3}$ This sentiment has recently been expressed by Jack Grubman, a well known telecom analyst with Salomon Smith Barney (in $[10])$.

${ }^{4}$ Investments by carriers such as Qwest Communications suggests that the cost may be lower.
} 
2. It is independent of content - once the channel has been allocated, it remains allocated whether it is used or not. That bandwidth cannot be used by others during idle periods.

3. Network attachments (i.e., telephones) are cheap because their functionality is specialized and limited. Numerous other characteristics also exist; the above list attempts to capture those of relevance to this study.

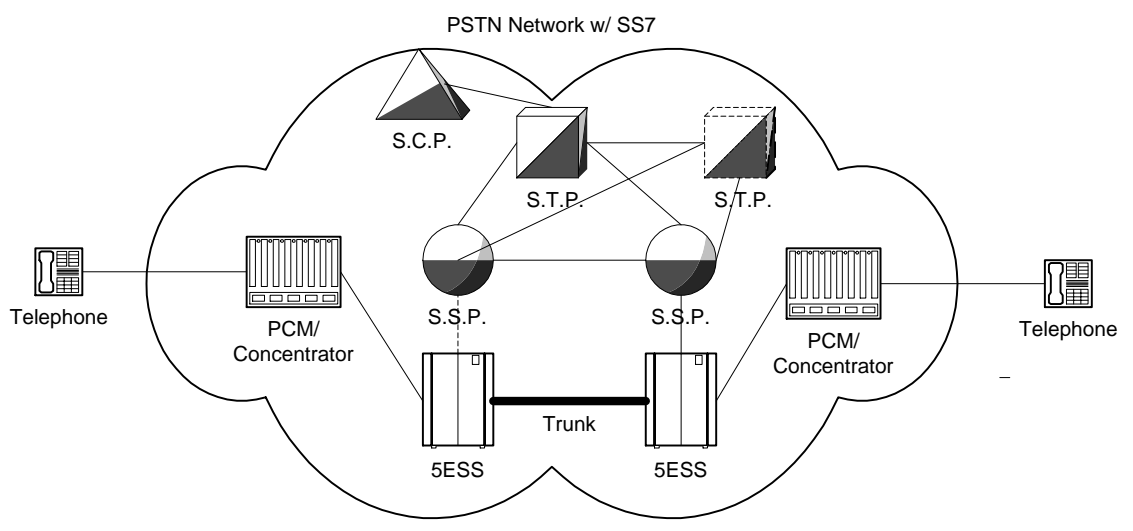

Figure 1: Circuit Switched Architecture

\subsection{Internet Telephony}

Internet telephony has grown as a specialized application of the Internet. A dominant characteristic of TCP/IP, as with most packet networks, is that most resources are shared (as opposed to dedicated). Thus, the bandwidth of a transmission channel is dynamically allocated to those who are using it at the moment. If their use disappears for a time, no system resources are dedicated to that user. The system was not designed to support services that require guaranteed timely packet arrivals. In summary, the essential characteristics of TCP/IP networks are:

1. It is capable of handling many application types, and allocating bandwidth dynamically between them on demand. This makes the development of integrated services particularly easy.

2. It cannot easily make performance guarantees, especially arrival time guarantees. This leads to quality of service degradation if the network is used for voice traffic. Note that this can be substantially mitigated if the network is engineered to low utilization, which increases cost.

\subsection{Architecture of Internet Telephony}

In this subsection, we discuss the different feature components of Itel from the circuit switched network. Followings are the key components of Itel architecture.

Loop Interface Since we assumed only that the copper wires in the loop were constant, we configured the Itel approach with xDSL. As a result, the Itel configuration includes DSLAMs, which raises the capital cost of Itel significantly. We also assume that the users have an Itel "appliance" ${ }^{5}$ that performs the G.729A compression and packetization using RTP/UDP/IP.

\footnotetext{
${ }^{5}$ See, for example, http://www.selsius.com: Selsius phone 12S Series' price is ranging from $\$ 200$ to $\$ 400$ currently.
} 


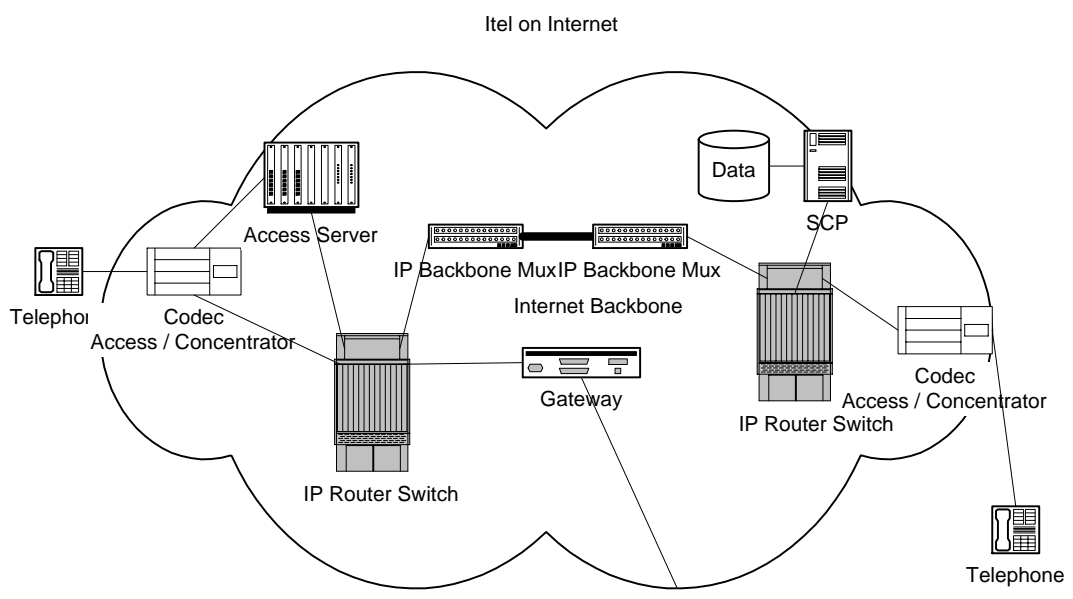

Figure 2: Internet Telephony Architecture

IP router switches IP router switches operates at the much higher performance than the traditional IP routers. It is also interoperable with the conventional IP routers and have faster packet processing and forwarding capability with the combination of layer- 2 switching and layer 3 routing. Many of recent IP switches provides packet processing rates of more than millions of packets per sec and internal switching bus more than Gbps speed. Some of the IP products support multiple upgradeable switching processors and Virtual Circuit (VC) layer 2 switching will enable the high speed switching bus to act like the fast space switching fabric. Implementation of IP switch is vendor dependent. In our simulation, we mainly assumed Cisco equipment (see [6] for more discussion).

Backbone OC-3 Trunk carrying IP IP over SONET technology is being implemented in many of IP backbone router switches. Some of the variant can be IP over ATM over SONET. IP over ATM over SONET solution might be attractive when the volume of traffic is integrated service and protocol based. However, if IP traffic becomes dominant as would be the case for Itel carriers, direct IP over SONET would be the more efficient solution [7]. The Itel model evaluated in this paper will already have 50 byte packets ( 40 bytes of RTP/UDP/IP overhead and 10 bytes for the voice payload) and the cell-based ATM transmission will increase significant amount of overhead again for transmission. Figure 6 shows the protocol stack of Itel central switching office example. In our model, typical OC-3 SONET trunks among COs provide enough capacity utilizing a utilization of less than $40 \%$ for most of the trunks.

Backbone Itel Gateway supporting SS7 In the case of Hybrid packet and circuit switched network would require the gateway solution to provide the signaling interface and routing. Numbering-to-IP conversion function may be implemented in this gateway.

\subsection{RTP/UDP/IP Protocol Stack in Itel}

The dominant standard for transmitting real time data in packet switched networks is ITU standard H.323 which uses RTP/UDP/IP encapsulation. RTP is a Real-Time Transport Protocol which supports end-to-end delivery services of applications transmitting real-time data over IP networks. RTP is defined in in RFC 1889 [3] and recommended to be used for audio and video in RFC 1890 [9]. RTP does not guarantee timely delivery or quality of service. RTP typically runs over UDP to utilize its multiplexing and checksum services. RTP provides the sequence number and time stamp information needed to assemble a real time data stream from packets. RTP also specifies the payload type to assign multiple data and compression types ${ }^{6}$.

\footnotetext{
${ }^{6}$ G.723, G.729, and G.729A are the popular compression types for the codecs in Voice Over IP (VIOP). The VOIP standard committee proposed a subset of H.323 for audio over IP. Many Internet Telephony vendors developed the products based on this standard.
} 


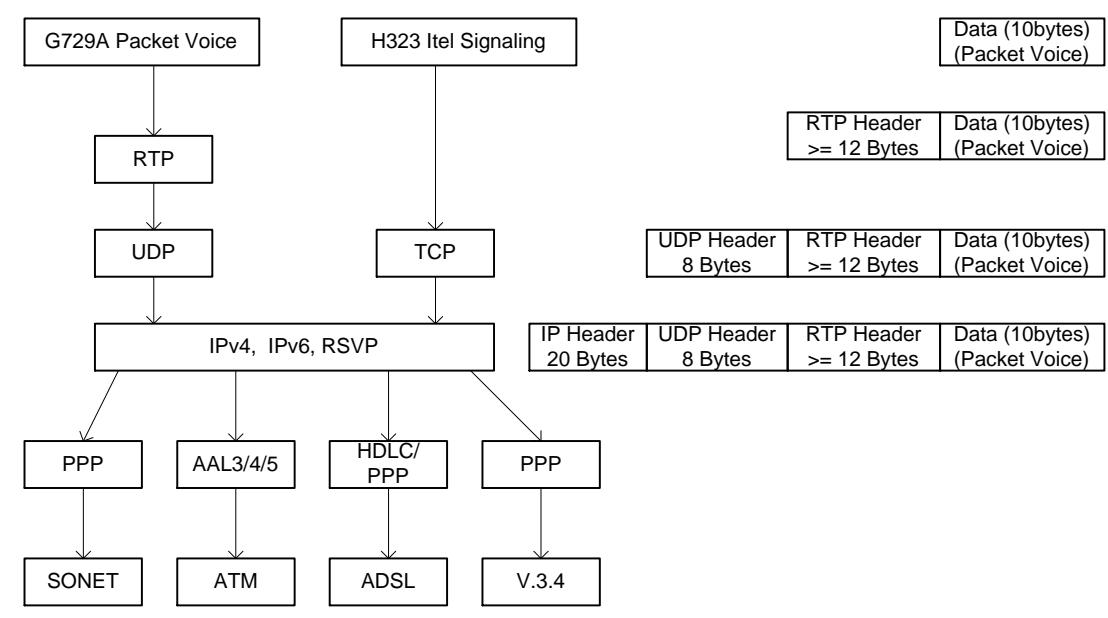

Figure 3: RTP protocol stack

\section{Model Description}

In this section, we describe the parameters of the simulation model. The network parameters for the circuit switched case and the Itel case are presented in their respective sections along with the summary results from the simulation.

\subsection{The Service Area}

To estimate the cost for each system, we constructed a "green field" system of each type for the same service area (a population of 1 million people uniformly distributed over an area of 3140 square kilometers equivalent to the U.S. State of Rhode Island). To simplify the calculation, we make the following assumptions:

- An average population density of 2.2 person per household

- Square service area (56km per side)

- No geographical barriers

- Households uniformly distributed over the service area (constant population density)

- Homogeneity of users

- Consistency of a user's behavior between systems (i.e., we assume away price and demand issues)

We assumed the local loop with with 19 gauge copper twisted pairs which can be extended up to $30 \mathrm{k}$-ft. The US's local loop standard specification is 22 gauge wire extending up to $5.5 \mathrm{~km}$ (1.8 k-feet) without load coil. Since we will assume all the loop conditions and requirement will be same, we will assume the situation with 19 gauge wire. We will assume five local switching location because 5ESS-2000 typically support up to 100,000 line and the number of switching location is agreeing with the local loop length. If we assume one line per household, then each local switching location ( CO or ITSP-CO) is terminating its assigned 454545 local loop lines. We assume all the originated traffic be distributed as $10 \%$ for outgoing from its service region and the rest for five local switching points evenly. The incoming traffic from the other service region is assume to be same with the amount of outgoing through one Tandem or NAP.

\subsection{Circuit Switched Model}

In addition to the general assumptions described above, we have made following additional assumptions that pertain specifically to the circuit switched network: 


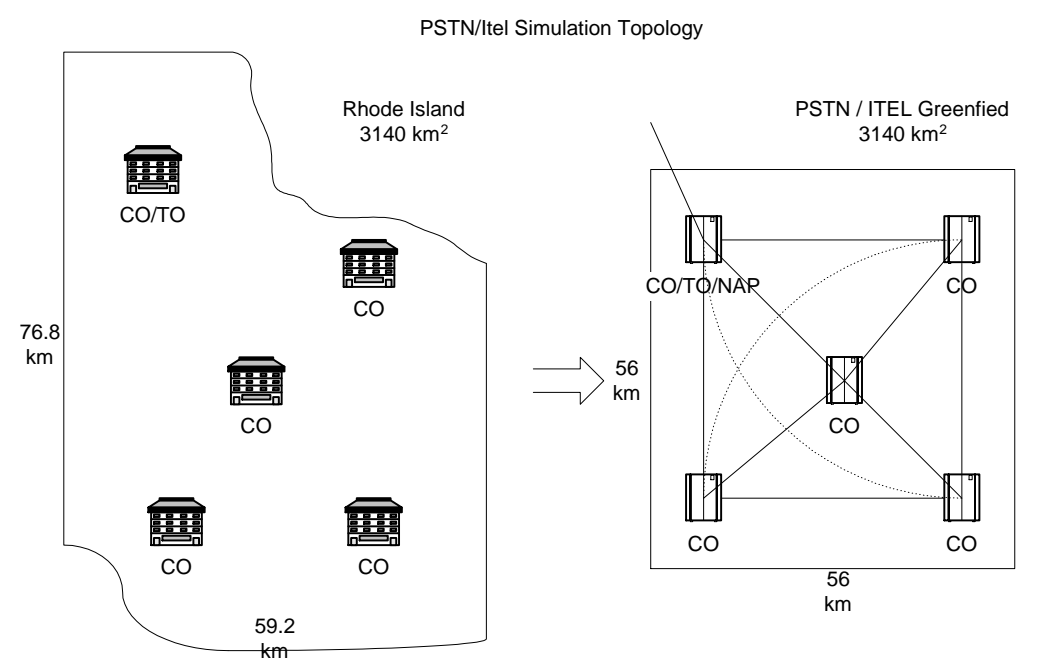

Figure 4: Simulation Topology

1. A 5ESS-2000 switch supports 100,000 lines at $3.6 \mathrm{CCS} /$ line (0.1 Erlang) in the busy hour. Therefore, each switch is receiving 9090.9 Erlangs of traffic from its assigned local service loops.

2. We assumed the most of the blocking occurs not in the switches but at the trunk side among the switches at one percent blocking probability.

3. Since we have only five switches within the given service region, we assumed each switch will have a direct trunk from and to each others.

4. The signaling network (SS7 Signaling System 7) is configured in a way that each local switch equipped SSP (Service Switching Points) connects to two STPs (Signal Transfer Points) and additional two STPs are forming a quadrupled mesh STP networks to access a SCP and outside signaling network STPs. Each signaling link (between SSP and STP) is engineered to have 0.4 utilization, so that if a failure occurred, the expected utilization would be 0.8 per link.[2]

5. The data rate per signaling link is $64 \mathrm{kbps}$. Each call generates average 3.5 signaling messages from an originating party and 3.5 signaling messages from the terminating party. Average signal length is 15 octet per message which comprise 1.875 msec duration.

The assumptions are summarized in Table 1.

Based on the above assumptions we dimensioned circuit switched network as follows:

1. The capacity of the trunks between any two normal COs and between a CO and Tandem will be bidirectional 210,816 Kbps (for each direction) and 326,400 Kbps respectively. With this configuration, COMNET III simulated 1 percent call blocking.

2. The capacity of the signaling links between a SSP and a Local STP; between two local STPs; between a Local STP and Remote STP and between two Remote STPs are bi-directional 326 Kbps (326 Kbps for each direction), 1,630 Kbps, $111 \mathrm{Kbps}$ and $222 \mathrm{Kbps}$ respectively.

The summary of circuit switched network simulation output is in Table 2. The simulated trunk capacity (211 Mbps) between each CO and Toll Office requires two OC- 3 and one OC-1 SONET leased trunks ${ }^{7}$. The trunk capacity (327 Mbps) between each CO requires one OC-3 and one OC-1 SONET Trunks ${ }^{8}$. The OC-1s shown in the table are the separate trunks between each $\mathrm{CO}$ which cannot be aggreagted to OC-3s.

\footnotetext{
${ }^{7}$ Total 4 pairs of this type are required in the simulated service area networks.

${ }^{8}$ Total 6 pairs of this type trunks are required in the simulated service area networks.
} 


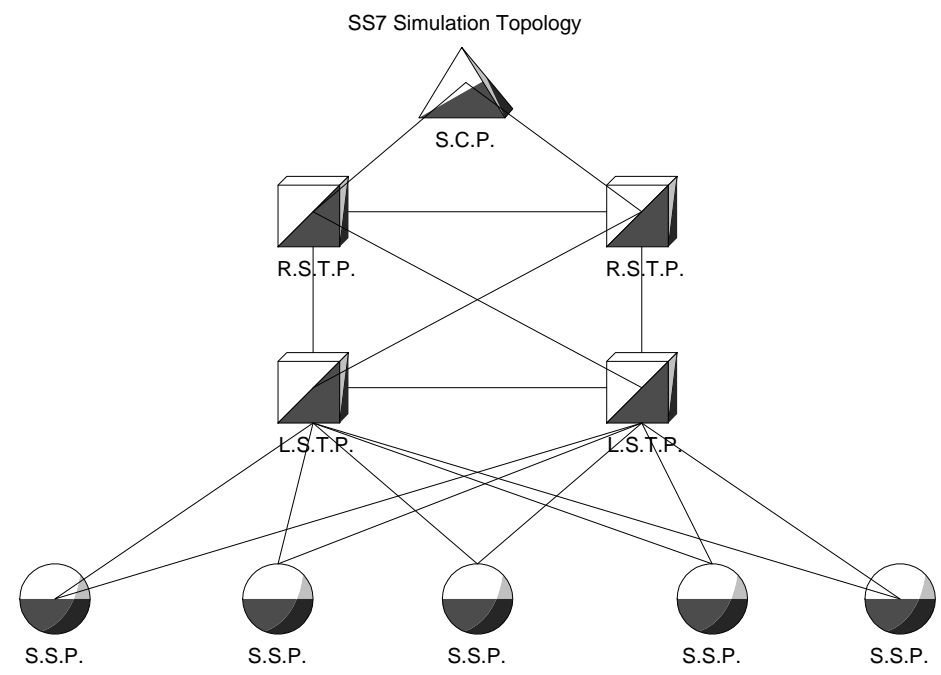

Figure 5: SS7 Network Simulation Topology

\begin{tabular}{|l|c|}
\hline \multicolumn{1}{|c|}{ Circuit Switched and SS7 Network Parameters } & Values \\
\hline \hline \multicolumn{1}{|c|}{ Ckt. Switched Parameters } \\
\hline Data Rate per Channel & $64 K b p s$ \\
Local Loop & 19 gauge twisted pair \\
Circuit Switch & 5ESS-2000 \\
Fraction of outgoing call & 0.1 \\
Originated Traffic per line & 0.1 Erlangs \\
No. of loop lines per CO & 90909 \\
Inter Arrival Time for CO & $0.0264 \mathrm{sec}$ \\
IAT for Toll Office & 0.0176 \\
Average Call Duration & $240 \mathrm{sec}$ \\
\hline \multicolumn{2}{|c|}{ SS7 Parameters } \\
\hline No. of SSP & 5 \\
No. of LSTP & 2 \\
No. of RSTP & 2 \\
No. of SCP & 1 \\
Engineering Utilization & 0.4 \\
Unit of SS7 link group & 8 DSOs \\
Average signaling messages per call & 7 \\
Average signaling message size & 15 octets \\
\hline
\end{tabular}

Table 1: Assumptions for Circuit Switched Model 


\begin{tabular}{|l|c|}
\hline Circuit Switch and SS7 Network requirement & Values \\
\hline \hline \multicolumn{2}{|c|}{ Circuit Switched Network } \\
\hline Four Trunks between each CO and a Toll & 4 X (2 OC-3 and 1 OC-1) \\
Six Trunks between each CO & 6 X (1 OC-3 and 1 OC-1) \\
Blocking Probability & 0.01 \\
Call Attempted per Hour & 750299 \\
Call Carried per Hour & 742454 \\
\hline \multicolumn{2}{|c|}{ SS7 Network } \\
\hline SS7 Links between SSP and LSTP & 80 DS0 \\
SS7 Links between LSTPs & 32 DS0 \\
SS7 Links between LSTP and RSTP & 8 DS0 \\
SS7 Links between RSTPs & 8 DS0 \\
\hline
\end{tabular}

Table 2: Summary of Simulation Output for Circuit Switched Network

\subsection{Itel Model}

Itel simulation model assumed in our analysis is based on RTP/UDP/IP standardized on ITU H.323. In this protocol, sequence numbers and time stamps are used to reassemble the real time voice traffic, although this provides no quality guarantee. TCP/IP is used to control the call (like SS7 in the circuit switched model). The simulated Itel model would provide the comparable functionality of network with circuit switched model, however it will not provide comparable quality and reliability of voice calls under circuit switching.

Figure 6 represents the Itel architecture model in our simulation. The simulation is concentrated on the IP router switching and trunking. IP Access Server and Edge Concentrator in the figure represent DLSAMs (Digital Subscriber Line Access Multiplexer) which do not have much effect on delay in the simulation.

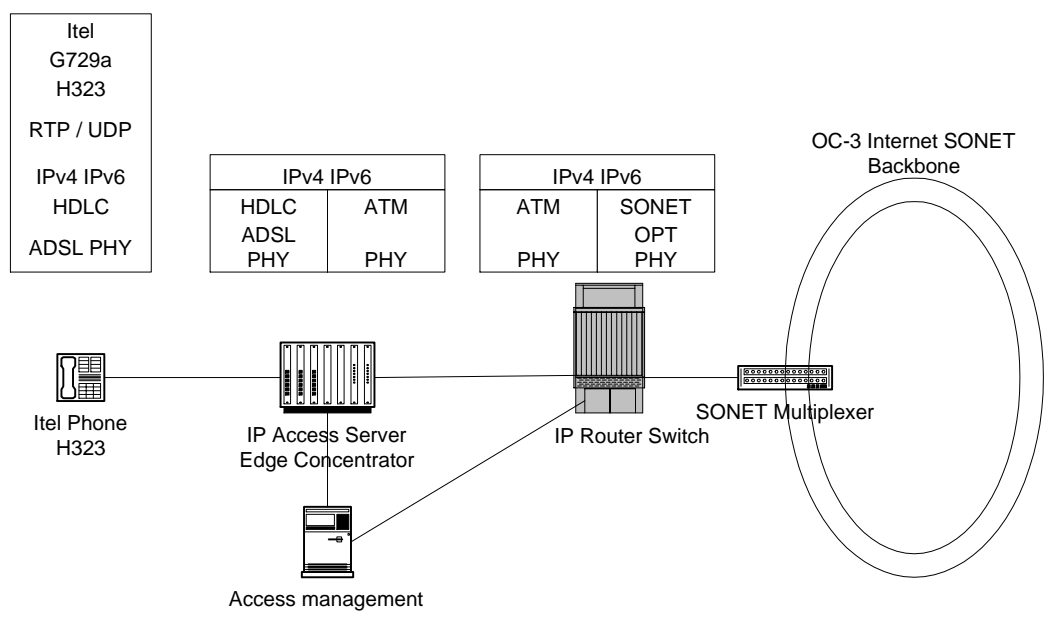

Figure 6: Internet Telephony Central Switching Office Protocol Stack

The assumptions made for Itel simulation model are specified below and in Table 3 .

- All voice traffic would be compressed from $64 \mathrm{Kbps}$ PCM voice to $8 \mathrm{Kbps}$ compressed data using G.729A codec.

- Silence suppression will be enabled in each codec, with $60 \%$ of a session being silent in one way.

- On the suppressed codec output, RTP, UDP and IP overhead will make actual average throughput around $14 \mathrm{Kbps}$. 
- Each voice is packetized every $10 \mathrm{msec}$ making 10 bytes payload through compression codec and enveloped in the 40 bytes RTP/UDP/IP header. The operational details of the RTP/UDP/IP is described in the previous Internet Telephony section.

- For the packet voice, the burst packet voice is modeled with average $350 \mathrm{msec}$ exponentially distributed active state and $650 \mathrm{msec}$ exponentially distributed silence state ${ }^{9}$.

- The Itel call is modeled as a connectionless UDP/IP session with exponentially distributed session length of $240 \mathrm{sec}$.

\begin{tabular}{|l|c|}
\hline Itel Network Parameters & Values \\
\hline \hline Compressed Peak Data Rate per Channel & 8 Kbps (G.729A) \\
Local Loop & 19 gauge twisted pair, ADSL \\
Packet Switch & IP Switch $(>10 \mathrm{Gbps})$ \\
Fraction of outgoing call & 0.1 \\
Originated Traffic per line & 0.1 Erlangs \\
No. of loop lines per CO & 90909 \\
Packet Voice Size & 10 bytes $(10 \mathrm{msec})$ \\
Protocol Overhead & 40 bytes (RTP/UDP $/ \mathrm{IP})$ \\
Packet Voice Burst Distribution & burst $350 \mathrm{msec}$, silence $650 \mathrm{msec}$ \\
Packet Delay Constraint & less than $250 \mathrm{msec}$ \\
RTP Session (Itel Call) Setup Delay Constraint & less than $1 \mathrm{sec}$ \\
\hline
\end{tabular}

Table 3: Assumptions for Itel Simulation Model

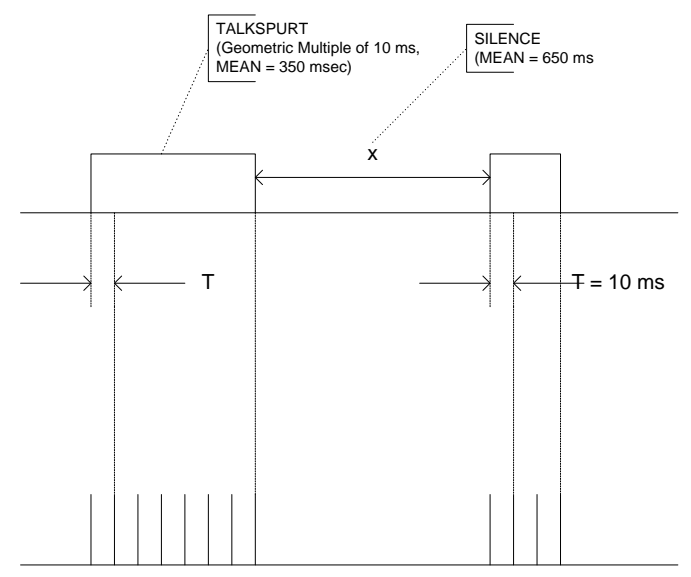

Figure 7: Packetized Voice Distribution

In the simulation, we found the average and worst case delays for packets through the network. This delay was modelled using the delay budgets in Table 5 . We assumed that this would provide a connection equality that was approximately equivalent to circuit switched voice ${ }^{10}$ (Table 4 shows the ITU recommendations). The source was initially modelled using an "on-off" speaker model. We found that, when aggregated, these could be modelled reasonably well by an exponential distribution, so that is what we used to reduce the running time of the simulation.

\footnotetext{
${ }^{9}$ See Figure 7.

${ }^{10}$ Total delay is a function of operating system and sound device delay in the end devices as well as network delay. Informal measurements suggest that these delays can be very high in WinTel PCs using standard sound cards.
} 


\begin{tabular}{|ll|}
\hline One Way Delay & Description \\
\hline \hline $0-150$ & Acceptable for most user applications. \\
\hline $150-400$ & $\begin{array}{l}\text { Acceptable provided that administrations are aware of the transmission } \\
\text { time impact on the transmission quality of user applications. } \\
\text { 400- }\end{array}$ \\
& $\begin{array}{l}\text { Unacceptable for general network planning purposes; however it is recognized } \\
\text { that in some exceptional cases this limits will be exceeded. }\end{array}$ \\
\hline
\end{tabular}

Table 4: ITU Delay Recommendations

\begin{tabular}{|l|c|c|}
\hline Itel Delay Component & Average Values & Maximum Values \\
\hline \hline \multicolumn{3}{|c|}{ Simulated Itel Delay } \\
(only switching process transmission and queueing delay) \\
\hline Packet Delay & $25 \mathrm{msec}$ & $179 \mathrm{msec}$ \\
RTP Session Setup Delay & $18.7 \mathrm{msec}$ & $91 \mathrm{msec}$ \\
\hline \multicolumn{3}{|c|}{ Deterministic Itel Delay } \\
\hline Encoding and Decoding G729A & $20 \mathrm{msec}$ & $20 \mathrm{msec}$ \\
Look Ahead Delay & $5 \mathrm{msec}$ & $5 \mathrm{Msec}$ \\
Itel Phone Processing Time & $100 \mathrm{msec}$ & $200 \mathrm{msec}$ \\
\hline Total & $150 \mathrm{msec}$ & $404 \mathrm{msec}$ \\
\hline
\end{tabular}

Table 5: Delay Budget for Itel Simulation Model

\begin{tabular}{|c|c|}
\hline Itel Network requirement & Values \\
\hline \multicolumn{2}{|c|}{ Itel Network } \\
\hline Trunk among COs & OC-3 Sonet Ring (= 10 OC-3) \\
\hline Local interface of each IP Switch & 12 DS-3 ATM \\
\hline Major Network Components & Itel Access Server \\
\hline & Concentrator \\
\hline & IP Switch \\
\hline & Sonet Mux \\
\hline \multirow{2}{*}{\multicolumn{2}{|c|}{$\begin{array}{c}\text { Itel Delay Budget } \\
\text { (only switching process transmission and queueing delay) }\end{array}$}} \\
\hline & \\
\hline Average Switch Utilization & 0.48 \\
\hline Maximum Switch Utilization & 0.6 \\
\hline Average Link Utilization & 0.43 \\
\hline Maximum Link Utilization & 0.59 \\
\hline Average Packet Delay & $25 \mathrm{msec}$ \\
\hline Maximum Packet Delay & $179 \mathrm{msec}$ \\
\hline Average Setup Delay & $18.7 \mathrm{msec}$ \\
\hline Maximum Setup Delay & $91 \mathrm{msec}$ \\
\hline \multicolumn{2}{|c|}{ Additional Itel Delay Consideration } \\
\hline Encoding and Decoding & $20 \mathrm{msec}$ \\
\hline Itel Phone Processing Time & $100 \mathrm{msec}$ \\
\hline
\end{tabular}

Table 6: Summary of Simulation Output for Itel 
Unlike the conventional ISP which is terminating the user line through CSU and DSU on LAN (10 Mbps or $100 \mathrm{Mbps}$ ), the Itel user access line for the carrier solution is terminated through a DSL Access Module (DSLAM), which is connected to the IP switches via internal OC3 or T3 circuits. Figure 6 shows the operational architecture of VoIP Itel Co/ISP model. The simulation results show that 12 DS3 interfaces is required for the local interface with the concentrator from IP switches and OC-3 line can support all trunk connection between any COs with more than $50 \%$ spare capacity. With OC-1, the trunks are so fully utilized that it icreases the packet delay critically in the simulation. Therefore one SONET OC-3 Ring would provide enough capacity for the assumed Itel trunk traffic among the ISP/COs. In this simulation model, SS7 call setup function is simulated as session setup using TCP/IP. Therefore, no additional capacity dimensioning is required for each links. The output of simulation for Itel is summarized in Table 6 .

\section{Estimated System Costs}

With this design in hand, we consulted with vendors (Cisco and Lucent) to review the "reasonableness" of the design and to estimate the cost of the switches/routers. The cost estimates we used were based on the information from Lucent (circuit switched network) and Cisco (Itel network). The cost of the transmission links is based on leased line costs from AT\&T.

The cost shown here is only focused on the differences of switching costs and trade-off trunking costs between circuit switching and packet technologies. We focused on the facility differences in the Central Offices.

The cost difference of the switching technology is composed of two parts; initial investment costs and yearly recurring costs. Most of the switching equipment in a $\mathrm{CO}$ will be considered the initial capital investment costs and transmission links (OC-3, etc.) will be considered the recurring costs. The life of telephone Central Office equipment can be assumed in several ways. According to IRS documents, the product life of telephone switching equipment (Class 48.12) is 18 years $^{11}$. Given the pace of technological change, we are doubtful that such long depreciationschedules will be sustainable in the future. Using the cost data, we have conducted sensitivity analysis of the monthly subscriber line costs in terms of the product life varying 3 years to 20 years and MARR (Minimum Attractive Rate of Return) ${ }^{12}$ ranging from $5 \%$ to $50 \%$.

\subsection{Circuit Switched Costs}

The simlation results indicate that a total of 14 OC-3's and 12 OC-1's would be needed, resulting in a $\$ 1.454$ million monthly cost of trunking. The bulk price with discount rate of $50 \%$ of the cost yields $\$ 727$ thousand per month. A 5ESS switching system costs around $\$ 500 \mathrm{~K}$ dollars (switching only) [10] ${ }^{13}$. Figure 8 is sensitivity analysis of monthly per line $\operatorname{costs}^{14}$. Our result shows that the total local switching cost is $\$ 2.5$ Million dollars (initial switching investment cost) and that the monthly cost (switching and trunking) per subscriber line is $\$ 1.7$ when the 5-year product life and 5\% MARR are assumed. $\$ 1.6$ out of $\$ 1.7$ comes from the cost of trunking per month ${ }^{15}$. The major cost factor of the circuit switched network is in trunking cost.

\subsection{Itel Costs}

For the Itel network, we used the same number of "CO's" so that the local loops between the two networks would be identical. While this might yield a sub-optimal Itel design, it was necessary so that we could ignore local loop costs in our analysis. The cost for Itel switching reached to the NPV of $\$ 28$ Million with high

\footnotetext{
${ }^{11}$ From IRS Publication 534, Depreciation

${ }^{12} \mathrm{MARR}$ is frequently used in the engineering economic analysis to represent the investers' expecting Rate of Return. Investors are using this MARR, instead of Interest Rate, when they convert the NPV of initial capital investment to the averaged recurring cost with their return over a given life time of the capital assests. MARR may vary depending on the developing pace and the riskiness of business and industry investors are involved or interested in.

${ }^{13}$ We are still awaiting final cost figures from Lucent. Given the overwhelmingly greater trunking costs, most plausible variances from this figure will not make that much difference.

${ }^{14}$ This is bottom line cost because we counted a 5ESS switch in all the CO locations and required leased trunks only.

${ }^{15}$ The figures shown here are the cost figures for the circuit switched network, which dose not include the access and signaling equipment. We will see this cost is still high enough to top the Itel cost analyzed in the section 4.2.
} 


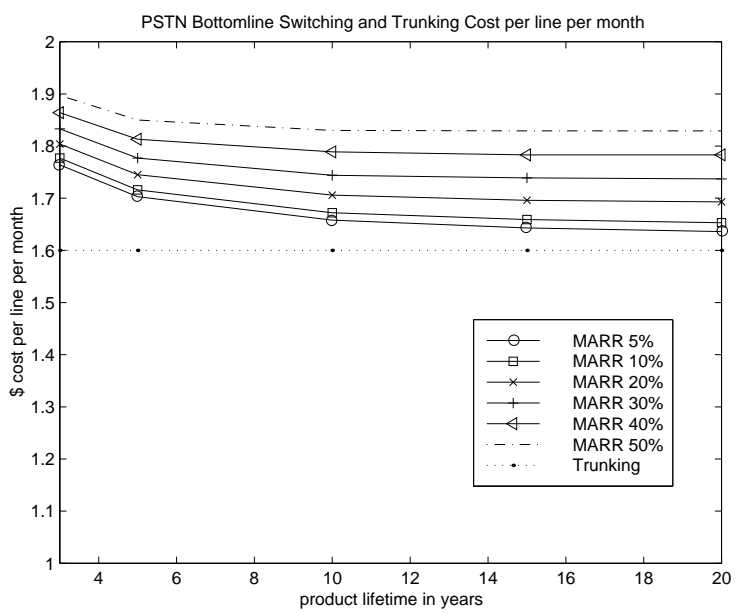

Figure 8: Circuit switched network cost per line per month

portion of DSLAM cost. The monthly discounted bulk rate trunking price is $\$ 286$ thousand dollars. The monthly Itel subscriber line cost for switching and trunking is $\$ 1.058$ when the 20-year product life and $20 \%$ MARR are assumed. As with circuit switched network, the dominant cost component is the trunking cost which comprises $\$ 0.63$ out of the $\$ 1.058$ monthly Itel subscriber line cost for switching and trunking. Figure 9 shows the cost per line per month for varying product lifetime and MARR. Since some of the price information is confidential, we only have shown the summarized figures in the paper.

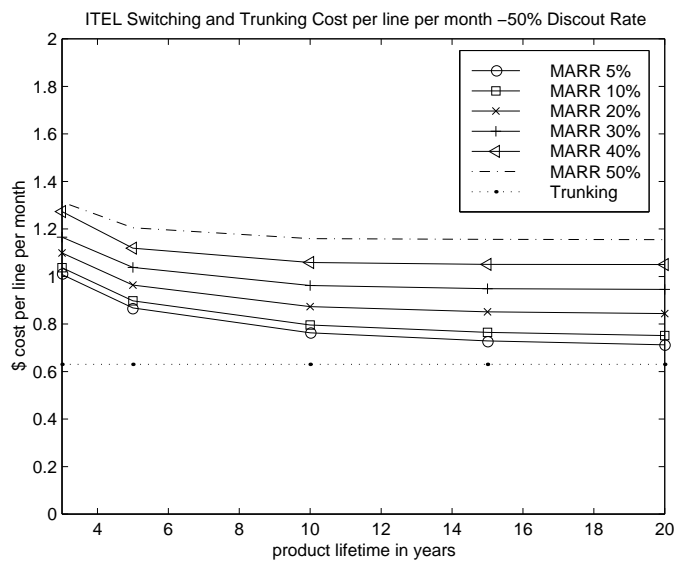

Figure 9: Itelcost per line per month-50\% Discount Rate for Switching Equips

\section{Discussion and Conclusions}

The results of this analysis shows that the interoffice transmission and switching costs for Itel approach are lower than for the circuit switched approach for a modestly sized circuit switched network by about $\$ 0.60$ per month (or $38 \%)^{16}$ using a $5 \%$ MARR and a capital equipment life of 10 years. The dominant cost factor is the cost of interoffice transmission; the compression and silence suppression enabled the use of $29 \%$ less

\footnotetext{
${ }^{16}$ This cost advantage obtained even though we used a more expensive access approach in xDSL than in the circuit switched network.
} 


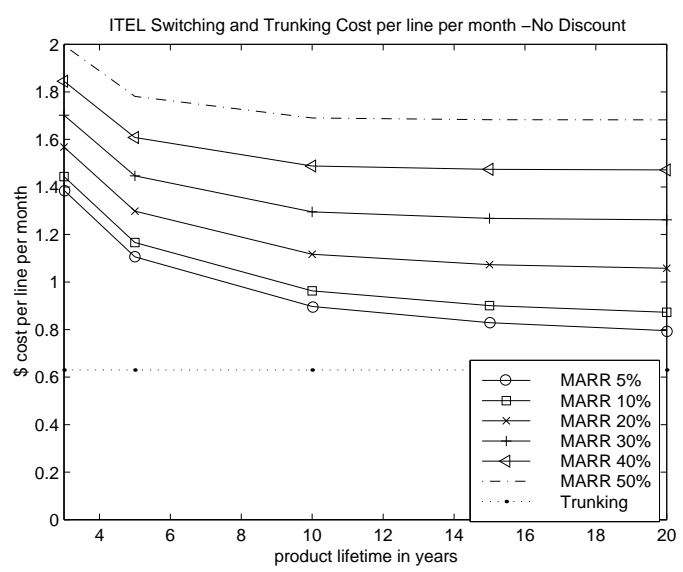

Figure 10: Itel cost per line per month-No Discount for Switching Equips

transmission capacity in Itel ${ }^{17}$. This suggests that the regulatory issues raised by Itel will not go away, and that regulators will have to continue to confront them. It also suggests an imminent technology conversion for telephone companies as they continue to seek lower costs of delivering their services.

Still, these results do need to be put into perspective. Generally speaking, the transmission and switching costs of an IXC are around $22 \%$ of their total cost structure [10]. One of the largest unknown costs for Itel are the OA\&M costs and billing $\operatorname{costs}^{18}$. These systems are highly developed for circuit switched networks and are of major importance to carriers. Finally, we did not consider operations cost differences between the two technologies, or their relative reliability, security etc. There is no reason to believe that these would be constant across the technologies.

Also, we did not account for terminal devices. The total system costs must include the cost of terminal equipment. At a high level, analog telephones are inexpensive, ranging in price from about $\$ 30$ to about $\$ 150$, which amounts to $\$ 0.50$ to $\$ 2.50$ per month over five years. Internet telephones are likely to be considerably more costly. Since the cost advantage of Itel is about $\$ 0.60$ per month, this suggest a break even of $\$ 1.10$ to $\$ 3.10$ per month, or $\$ 66$ to $\$ 186$ in total capital cost, ceteris paribus. If these are extensions to PCs, then these costs would have to be the incremental capital costs for providing Itel over an existing platform.

Another important factor is the ability to deliver integrated services. We did not consider the incremental cost of developing and deploying integrated services in both networks. The relative cost of this (and the relative revenue opportunities based on the capabilities of the terminal device) could be an important factor in determining a carrier's choice of switching and transmission technology.

\section{References}

[1] Barbetta, F. Can carriers find a business case? Business Communications Review (May 1998), $24-30$.

[2] Bellcore. Technical reference on SS7. TR-NWT-000082, 1987.

[3] Casner, S., Frederick, R., Jacobsen, V., and Schulzrinne, H. RTP: A transport protocol for real time applications. ftp://ftp.isi.edu/in-notes/rfc1899.txt, January 251996.

[4] Clark, D. D. A taxaonomy of internet telephony applications. Internet Telephony Consortium (1997), 51.

\footnotetext{
${ }^{17}$ The Itel network required 10 OC-3s vs. the 14 required for circuit switching.

${ }^{18}$ Based on informal conversations with a representative from Hyperion Communications.
} 
[5] Frieden, R. Dialing for dollars: Will the FCC regulate Internet telephony? Telecommunications Policy Research Conference, 1997.

[6] Kumar, V. P., Lakshman, T., and Stiliadis, D. Beyond best effort: Router architectures for the differentiated services of tomorrow's internet. IEEE Communications Magazine (May 1998), 152-164.

[7] Manchester, J., Anderson, J., Doshi, B., and Dravida, S. IP over SOnet. IEeE Communications Magazine (May 1998), 136-142.

[8] Mueller, M. L. Universal Service. AEI Studies in Telecommunications Deregulation. MIT Press, Cambridge MA, 1997. ISBN 0-262-13327-X.

[9] Schulzrinne, H. RTP profile for audio and video conferences with minimal control. ftp://ftp.isi.edu/innotes/rfc1890.txt, January 251994.

[10] Stuck, B., And Weingarten, M. Can carriers make money on IP telephony? Business Communications Review (August 1998), 39-54. 